\title{
Polycyclic aromatic hydrocarbon exposure, obesity and childhood asthma in an urban cohort
}

\author{
Kyung Hwa Jung ${ }^{a}$, Matthew Perzanowski ${ }^{b}$, Andrew Rundle ${ }^{c}$, Kathleen Moors ${ }^{a}$, \\ Beizhan Yan ${ }^{\mathrm{d}}$, Steven N. Chillrud ${ }^{\mathrm{d}}$, Robin Whyatt ${ }^{\mathrm{b}}$, David Camann ${ }^{\mathrm{e}}$, Frederica P. Perera ${ }^{\mathrm{b}}$, \\ Rachel L. Miller ${ }^{\text {a,b,f,* }}$ \\ a Division of Pulmonary, Allergy and Critical Care of Medicine, Department of Medicine, College of Physicians and Surgeons, Columbia University, PH8E-101, \\ 630 W. 168th Street, New York, NY 10032, United States \\ ${ }^{\mathrm{b}}$ Mailman School of Public Health, Department of Environmental Health Sciences, Columbia University, 722 W. 168 Street, New York, NY 10032, United States \\ ${ }^{c}$ Mailman School of Public Health, Department of Epidemiology, Columbia University, 722 W. 168 Street, New York, NY 10032, United States \\ d Lamont-Doherty Earth Observatory, Columbia University, 61 Rt, 9W Palisades, New York 10964, United States \\ ${ }^{\text {e } C h e m i s t r y ~ a n d ~ C h e m i c a l ~ E n g i n e e r i n g ~ D i v i s i o n, ~ S o u t h w e s t ~ R e s e a r c h ~ I n s t i t u t e, ~} 6220$ Culebra Road, San Antonio, TX 78228, United States \\ ${ }^{\mathrm{f}}$ Division of Pediatric Allergy and Immunology, Department of Pediatrics, College of Physicians and Surgeons, Columbia University, PH8E-101, \\ 630 W. 168 Street, New York, NY 10032, United States
}

\section{A R T I C L E I N F O}

Article history:

Received 25 April 2013

Received in revised form

8 November 2013

Accepted 4 December 2013

Available online 27 December 2013

Keywords:

Childhood obesity

Methylphenanthrenes

Polycyclic aromatic hydrocarbons

Asthma

Nonatopic children

\begin{abstract}
A B S T R A C T
Background: Exposure to traffic-related air pollutants, including polycyclic aromatic hydrocarbons (PAHs) from traffic emissions and other combustion sources, and childhood obesity, have been implicated as risk factors for developing asthma. However, the interaction between these two on asthma among young urban children has not been studied previously.

Methods: Exposure to early childhood PAHs was measured by two week residential indoor monitoring at age 5-6 years in the Columbia Center for Children's Environmental Health birth cohort $(n=311)$. Semivolatile [e.g., methylphenanthrenes] and nonvolatile [e.g., benzo(a)pyrene] PAHs were monitored. Obesity at age 5 was defined as a body mass index (BMI) greater than or equal to the 95th percentile of the year 2000 age- and sex-specific growth charts (Center for Disease Control). Current asthma and recent wheeze at ages 5 and 7 were determined by validated questionnaires. Data were analyzed using a modified Poisson regression in generalized estimating equations (GEE) to estimate relative risks (RR), after adjusting for potential covariates.

Results: Neither PAH concentrations or obesity had a main effect on asthma or recent wheeze. In models stratified by presence/absence of obesity, a significant positive association was observed between an interquartile range (IQR) increase in natural log-transformed 1-methylphenanthrene (RR [95\% CI]: 2.62 [1.17-5.88] with $\left.\mathrm{IQR}_{\ln }=0.76\right)$, and 9-methylphenanthrene (2.92 [1.09-7.82] with $\left.\mathrm{IQR}_{\ln }=0.73\right)$ concentrations and asthma in obese children $(n=63)$. No association in non-obese $(n=248)$ children was observed at age $5\left(P_{\text {interaction }}<0.03\right)$. Similar associations were observed for 3 -methylphenanthrene, 9-methylphenanthrene, and 3,6-dimethylphenanthrene at age 7.

Conclusions: Obese young children may be more likely to develop asthma in association with greater exposure to PAHs, and methylphenanthrenes in particular, than non-obese children.
\end{abstract}

(c) 2013 Elsevier Inc. All rights reserved.

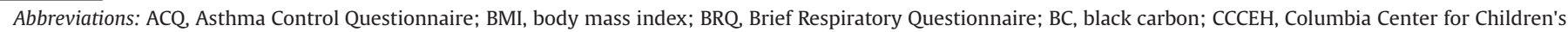

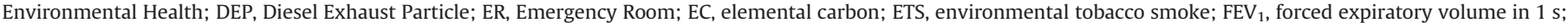
ICS, inhaled corticosteroids; IQR, interquartile range; ISAAC, International Study of Asthma and Allergies in Childhood; $\mathrm{NO}_{2}$, nitrogen dioxide; NYC, New York City;

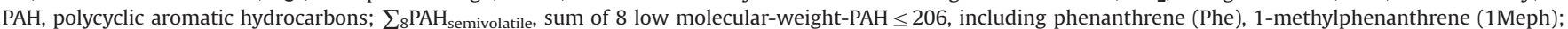
2-methylphenanthrene (2Meph), 3-methylphenanthrene (3Meph), 9-methylphenanthrene (9Meph), 1,7-dimethylphenanthrene (1,7DMeph), 3,6-dimethylphenanthrene

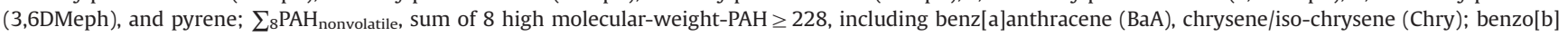
fluoranthene (BbFA), benzo[k]fluoranthene (BkFA), benzo[a]pyrene (BaP), indeno[1,2,3-c,d]pyrene (IP), dibenz[a,h]anthracene (DahA), and benzo[g,h,i]perylene (BghiP); PM, particulate matter; PUF, Polyurethane Foam; RR, relative risk

* Corresponding author at: Division of Pulmonary, Allergy and Critical Care of Medicine, Department of Medicine, College of Physicians and Surgeons, Columbia University, PH8E-101, 630 W. 168 Street, New York, NY 10032, United States. Fax: +1 2123052277.

E-mail addresses: kj2237@columbia.edu (K.H. Jung), mp2217@columbia.edu (M. Perzanowski), agr3@columbia.edu (A. Rundle), moorsk@gmail.com (K. Moors), yanbz@ldeo.columbia.edu (B. Yan), chilli@ldeo.columbia.edu (S.N. Chillrud),rmw5@columbia.edu (R. Whyatt), david.camann@swri.org (D. Camann), fpp1@columbia.edu (F.P. Perera), rlm14@columbia.edu (R.L. Miller).
} 


\section{Introduction}

The prevalence of childhood obesity has increased from $7 \%$ to $20 \%$ from 1980-2008 in the United States, and is higher for Hispanics (31\%) and Blacks (23\%) in New York City (NYC) (Thorpe et al., 2004). Concomitant with the rapid rise in childhood obesity, the prevalence of childhood asthma also has increased from $3.6 \%$ to 9.6\% during 1980-2009 (Akinbami et al., 2009; CDC, 2011), especially in urban areas like NYC (Nicholas et al., 2005).

Exposure to air pollution, and polycyclic aromatic hydrocarbons (PAHs) in particular, appears to be a risk factor for the development of asthma in urban settings (Jung et al., 2012b; Miller et al., 2004). For example, our previous study reported that repeated high exposure to $\mathrm{PAHs}$, semivolatile pyrene specifically, during prenatal period and age 5-6 years, is associated with asthma in young children (Jung et al., 2012b). Obesity also has been associated with the development of childhood asthma in both cross-sectional and prospective studies (Castro-Rodriguez et al., 2001; Gilliland et al., 2003; Visness et al., 2010). Further, the asthma-obesity association may vary by sex or atopic status (Castro-Rodriguez et al., 2001; Gilliland et al., 2003; Visness et al., 2010). For example, a study in the Tucson, Arizona cohort found that becoming overweight or obese between the ages of 6 and 11 years increased the risk of developing new wheeze at age 11 or 13 among girls but not boys (Castro-Rodriguez et al., 2001). However, a subsequent prospective study of school-age children in South California found that being overweight or obese was associated with an increased risk of incident asthma in boys, but not in girls; the strongest effect was observed among nonatopic boys (Gilliland et al., 2003). One study using the National Health and Nutrition Examination Survey (NHANES) data set (1999-2006) also found that obesity was associated with current asthma among children ages $2-19$, with a stronger association in nonatopic children (Visness et al., 2010).

Emerging evidence suggests that the presence of obesity may modify the effects of exposure to air pollution on respiratory disease. For example, obese individuals experienced a greater decline in forced expiratory volume in $1 \mathrm{~s}\left(\mathrm{FEV}_{1}\right)$ following ozone $\left(\mathrm{O}_{3}\right)$ exposure, compared to nonobese subjects (Alexeeff et al., 2007). This finding was supported by animal studies showing that genetically $(\mathrm{db} / \mathrm{db}$ mice lacking leptin receptor) or diet-induced obese mice exhibited increased airway hyperresponsiveness (AHR) and enhanced $\mathrm{O}_{3}$ induced pulmonary inflammation (e.g., greater interleukin (IL)-6 production) compared to nonobese mice (Johnston et al., 2008; Lu et al., 2006). These results suggest that obesity may confer greater vulnerability in airways following exposure to some air pollutants, yet the role of obesity following exposure to PAH on childhood asthma has not been studied previously.

PAHs have 2 types of anthropogenic sources: pyrogenic (i.e., incomplete combustion of organic material such as from traffic emissions and heating oil combustion) and petrogenic (e.g., unburned fossil organic material contribution such as direct evaporations from petroleum products). Alkylated PAHs (e.g., methylphenanthrenes), as opposed to parent PAHs (e.g., phenanthrene), are emitted more abundantly from petrogenic than pyrogenic sources (Saha et al., 2009). The toxicities of the individual phenanthrenes and methylaphenanthrenes during in vivo experiments are known to differ (Wolinska et al., 2011). While our earlier study suggests that repeated high exposure to unsubstituted semivolatile PAHs (i.e., pyrene) may induce asthma (Jung et al., 2012b), our understanding of the effects of exposure to alkylated PAHs (e.g., methylphenanthrenes) on respiratory health is more limited.

The goal of this study was to examine whether obesity may modify the effects of age 5-6 years PAH exposure, and semivolatile and alkylated PAHs in particular, on asthma in 5-7 years old innercity children living in Northern Manhattan and the Bronx. Under the auspices of the Columbia Center for Children's Environmental Health (CCCEH) birth cohort, we were able to control for prenatal
PAH concentrations and exposure to other indoor air pollutants such as fine particulate matter $\left(\mathrm{PM}_{2.5}\right)$ and soot/black carbon (BC). We hypothesized that obese children would be at greater risk of having asthma and recent wheeze with exposure to higher $\mathrm{PAH}$ than would non-obese children.

\section{Methods}

\subsection{Study population}

727 healthy and nonsmoking Dominican or African American mothers who reside in Northern Manhattan or South Bronx were enrolled into the CCCEH cohort during pregnancy (Miller et al., 2004). Questionnaires were administrated to the mother prenatally, every 3 months through age 2 years, and every 6 months thereafter. PAH concentrations were measured from residential indoor monitors for 408 of their children at age 5-6 years. Data were analyzed for those children $(n=363)$ for whom measures of indoor PAH measures at age 5-6, height and weight, and complete age 5 questionnaire data were available. The study was approved by the Columbia University Institutional Review Board and written informed consent was obtained from all study participants.

\subsection{Air monitoring}

Details of residential air monitoring have been published previously (Jung et al., 2010a). Indoor air monitors were placed in a room where the child spent most of his or her time for two weeks between 2005 and 2011 at age 5 through 6. Sixteen 3-ring to 6-ring $\mathrm{PAH}$ (eight semivolatile $(178 \leq \mathrm{MW} \leq 206)$ and eight nonvolatile $(228 \leq \mathrm{MW} \leq 278) \mathrm{PAH}$; see Supplementary material) were selected as target compounds due to their abundance in traffic emissions and their possible carcinogenicity and mutagenicity (Jung et al., 2010b). Nine of these PAHs (eight nonvolatile PAHs and pyrene) also had been measured prenatally using 48-hr personal air monitor-

ing between 1998 and 2006 (Miller et al., 2004). A single soxhlet extraction of both the filter and PUF together was analyzed for PAHs at Southwest Research Institute as described (Jung et al., 2010a, 2010b; Miller et al., 2004).

Residential indoor levels of $\mathrm{PM}_{2.5}$ and $\mathrm{BC}$ were measured simultaneously with PAH monitoring, with a repeat sampling 6 months later. Details of analytical protocols for $\mathrm{PM}_{2.5}$ and $\mathrm{BC}$ are described in Jung et al. (2010a, 2010b).

\subsection{Obesity}

Child's height and weight were measured at age 5, using a SECA wall-mounted stadiometer (SECA, Hamburg, Germany) and a Detecto Cardinal 750 digital scale (Cardinal Scale Manufacturing Company, Webby City, Missouri), respectively (Rundle et al., 2012). Children's body mass index (BMI; defined as weight in kilograms divided by height in meters squared $\left.\left(\mathrm{kg} / \mathrm{m}^{2}\right)\right) z$ scores and percentiles were calculated using the Centers for Disease Control (CDC) and Prevention SAS macro (Prevention, 2004). A child was classified as obese if the BMI was greater than or equal to the age- and sexspecific 95th percentile of the year 2000 CDC growth charts.

\subsection{Health outcomes}

The Brief Respiratory Questionnaires (BRQ) (Bonner et al., 2006) was used to assess current asthma, defined as parental report of doctor diagnosis of ever asthma AND a report of asthma medication use in the past 12 months. International Study of Asthma and Allergies in Childhood questionnaires (ISAAC) (Jenkins et al. 1996) was used to assess recent wheeze, defined as any report of wheeze in the last 12 months.

\subsection{Seroatopy}

Total and specific immunoglobulin (Ig)E to cat, mouse, dog, Dermatophagoides farinae and German cockroach at age 5 were measured in duplicate from sera using Immunocap (Phadia, Uppsala, Sweden) as described (Donohue et al., 2008). Children were considered sensitized to indoor allergens if they had a specific $\operatorname{IgE} \geq 0.35 \mathrm{IU} / \mathrm{mL}$ to any of the allergens tested. They were classified as having any allergic sensitization if total $\mathrm{IgE} \geq 50 \mathrm{IU} / \mathrm{mL}$. Children were defined as seroatopic if they met either of these criteria.

\subsection{Statistical analysis}

Age 5-6 years PAH were natural log-transformed to normalize skewed distributions (e-Fig. 1). As reported previously (Jung et al., 2010a), PAH were analyzed as individual $\mathrm{PAH}$, the sum of eight semivolatile $\mathrm{PAH}\left(\sum_{8} \mathrm{PAH}_{\text {semivolatile }}\right)$, and the sum of 
8 nonvolatile $\mathrm{PAH}\left(\Sigma_{8} \mathrm{PAH}_{\text {nonvolatile }}\right)$ due to the absence of correlations between semivolatile and nonvolatile PAH (e-Table 1). Relative risks (RR) are presented for an interquartile range (IQR) increase in each natural log-transformed PAH concentration. The associations among levels of age 5-6 years PAH, obesity and respiratory outcomes were analyzed in several ways using a modified Poisson regression in generalized estimating equations (GEE) to estimate RR (Zou, 2004). First, the models were adjusted for common potential covariates, including maternal race/ethnicity, sex, maternal asthma, maternal education, prenatal environmental tobacco smoke (ETS) exposure, any reported postnatal ETS exposure at 6, 12, 24, 36, and 60 months, and cold/influenza season. Dichotomous variables indicating seroatopic to indoor allergens, whether the residential monitoring was conducted at age 5 vs. 6 years, and season of childhood PAH measurement (heating season [October-April] vs. nonheating season [May-September]) also were controlled in the model. Further, natura log-transformed prenatal measures of $\mathrm{PAH}$, that were minimally or not correlated with measures of PAH at age 5-6 years, and natural log of the average of repeat indoor measurements of $\mathrm{PM}_{25}$ and $\mathrm{BC}$ were included in the model (adjusted model). Second, the subjects were stratified by obesity status and the adjusted models were run within each stratum (stratified model). Third, a multiplicative interaction term was included in the adjusted models (interaction model) to test whether the association between age 5 and 6 years PAH concentration and respiratory outcomes significantly varied by obesity status. The relative excess risk due to interaction (RERI) was estimated further by testing a departure from additivity with its 95\% confidence intervals from percentiles of 10,000 bootstrap samples.

Secondary analyses were conducted with the tertiles of age 5-6 PAH concentrations (the second [middle] and third [highest] vs. the first [lowest] as reference). Additional analyses were conducted to test the multiplicative interaction term for effect modification by obesity after stratifying by seroatopy and sex.

Sensitivity analyses were conducted as follows: (1) reanalysis after excluding children $(n=81)$ with an existing reported doctor diagnosed asthma at 36,48 , or 54 months, (2) reanalysis after substituting overweight (BMI $\geq 85$ th percentile) for obese and using BMI $z$ score, (3) reanalysis after controlling for pre-pregnancy maternal BMI, and (4) reanalysis after eliminating upper or lower 5\% of age 5-6 years PAH measures or removing children $(n=19)$ who took inhaled corticosteroids (ICS) in the last 3 months. All analyses were performed using PASW Statistic version 18.0 (SPSS Inc. Chicago, IL, USA) where $p<0.05$ was considered statistically significant.

\section{Results}

\subsection{Cohort characteristics}

Children who had residential air monitoring, and BMI data available, and completed questionnaires at age 5 did not differ demographically from CCCEH children excluded from the current analyses (i.e, missing residential measures, BMI or health outcome data due to age-related inclusion criteria; Table 1), with the exception of a slightly higher frequency of any allergic sensitization (i.e. total IgE $\geq 50 \mathrm{IU} / \mathrm{mL}\left[\chi^{2}\right.$ test; $\left.\left.p=0.02\right]\right)$. Seventy two percent (224/ 311) of residential air samples were collected at age 5 instead of age 6 (Table 1). Of the 408 children with PAH measurements, 97 children were dropped from the analysis due to missing covariates or invalid air pollution data, resulting in a final sample of 311 (Fig. 1).

\subsection{Age 5-6 years PAH concentrations, obesity, and asthma}

Descriptive statistics of age 5-6 years PAH concentrations are shown in e-Table 2, with $100 \%$ above limit of detection (LOD) of semivolatile PAH. Phenanthrene, the most abundant compound among the measured individual PAH (72\% of total sum of $16 \mathrm{PAH}$; e-Table 2) correlated strongly with concentrations of all other semivolatile PAH (e-Table 1. Pearson correlation $0.46 \leq r \leq 0.78$ ) but not with $\Sigma_{8} \mathrm{PAH}_{\text {nonvolatile }}(r=0.06)$.

Levels of $\Sigma_{8} \mathrm{PAH}_{\text {nonvolatile }}$ or semivolatile PAH alone were not associated directly with asthma or recent wheeze in adjusted models (Tables 2, $p>0.05$ ), consistent with our previous findings (Jung et al., 2012b; Miller et al., 2004). Also, in adjusted models childhood obesity was not significantly associated with asthma or wheeze $(p>0.05)$.

\subsection{Effect modification of the association between age 5-6 years PAH concentrations and asthma by obesity}

Among 63 obese children, higher levels of 1-methylphenanthrene (Fig. 2; RR [95\% CI]: 2.62 [1.17-5.88]), 9-methylphenanthrene (2.17 [1.06-4.45]), and 3,6-dimethylphenanthrene (2.69 [1.21-5.94]) were associated with asthma at age 5 years, after controlling for common covariates and air pollutants (prenatal $\mathrm{PAH}$, age 5-6 years $\mathrm{PM}_{2.5}$ and $\mathrm{BC}$ measures). These findings were not replicated when examining the associations between the other semivolatile $\mathrm{PAH}$ or $\Sigma_{8} \mathrm{PAH}_{\text {nonvolatile }}$ and asthma or wheeze among obese children (Table 2; data not shown for wheeze). Among 248 non-obese children, significant associations were not found between any PAH levels and asthma and wheeze (Table 2; data not shown for wheeze).

In the interaction model, a significant interaction between several semivolatile PAH levels (i.e., 1-methylphenanthrene, 9-methylphenanthrene and pyrene) and obesity on asthma at age 5 was found (Table 2, $P_{\text {interaction }}<0.03$ ). Consistently, a significantly greater than additive interaction was observed using the RERI (Supplementary material). In comparison, obesity showed no effect modification of the association between any PAH and recent wheeze (all $P_{\text {interaction }}>0.05$; data not shown).

Secondary analyses were conducted to assess the stability of the associations between age 5-6 years PAH exposure, obesity and asthma at age 7. Higher levels of 3-methylphenanthrene (Fig. 3, RR [95\% CI]: 3.25 [1.45-7.27]), 9-methylphenanthrene (5.12 [1.87-14.1]), and 3,6-dimethylphenanthrene (3.28 [1.40-7.71]) were associated with asthma in obese, but not non-obese 7 years olds $\left(P_{\text {interaction }}=0.01\right.$, $P_{\text {interaction }}<0.001$ and $P_{\text {interaction }}=0.005$ for 3-methylphenanthrene, 9-methylphenanthrene and 3.6-dimethylphenanthrene, respectively). Similar associations were found when PAH levels were categorized by tertiles. Among obese, but not non-obese children, the highest tertiles of 9-methylphenanthrene and 3,6-dimethylphenanthrene, as compared with the lowest, were significantly associated with asthma at both ages 5 and 7 years (e-Table 3; data not shown for age 7). The second tertiles, but not the third tertiles of phenanthrene, 1-methylphenenthrene, 2-methylphenanthrene, and $\Sigma_{8} \mathrm{PAH}_{\text {semivolatile }}$ were associated with higher relative risks of asthma at age 5 years (eTable 3).

In addition, to explore whether these associations vary by asthma phenotype (e.g., seroatopic vs. nonatopic) or sex (girls vs. boys), interaction analyses were repeated following stratification by seroatopy (elevated indoor specific allergen or total IgE) and sex. Among the non-seroatopic children, the interaction between obesity and all the semivolatile PAH except 1,7-dimethylphenanthrene became significant or borderline significant for asthma and wheeze (e-Table 4; $P_{\text {interaction }}<0.05$ for all). In addition, a significant positive interaction was found between 1-methylphenanthrenes and 1,7-dimethylphenanthrene and obesity on asthma in girls, but not boys (e-Table 5; $P_{\text {interaction }}<0.01$ ).

\subsection{Sensitivity analyses}

First, a significant interaction was replicated for all PAH, except pyrene and $\Sigma_{8} \mathrm{PAH}_{\text {nonvolatile }}$ at age 5 when the analyses were restricted to those without a preexisting doctor diagnosed asthma at 36,48 or 54 months (e-Table 6; $P_{\text {interaction }}<0.05$ ). Second, analyses for interactions were repeated for age 5-6 PAHs using overweight, defined as $\mathrm{BMI} \geq 85$ th percentile, or using a continuous BMI $z$ score. The interaction between phenanthrene and overweight reached significance for asthma $\left(P_{\text {interaction }}=0.03\right.$ ), while the interactions for other semivolatile $\mathrm{PAH}$ were not statistically significant. When obesity was replaced with BMI $z$ score, a significant interaction was replicated for all PAH, except 1,7-dimethlyphenanthrene, pyrene and $\Sigma_{8} \mathrm{PAH}_{\text {nonvolatile }}$ at ages 5 and 7 years (Table 3). After adjustment for maternal BMI, the 
Table 1

Demographic characteristic of the study cohort [No. (\%)] .

\begin{tabular}{|c|c|c|}
\hline \multirow[t]{2}{*}{ Characteristic } & Subjects included $^{\mathrm{b}} N=311$ & Subjects excluded ${ }^{\mathrm{c}} N=416$ \\
\hline & $N /$ total [\%] & $N /$ total $[\%]$ \\
\hline \multicolumn{3}{|l|}{ Maternal ethnicity } \\
\hline Dominican & $193 / 311[62]$ & $280 / 416$ [67] \\
\hline African-American & $118 / 311[38]$ & $136 / 416[33]$ \\
\hline Girls & $164 / 311[53]$ & $212 / 416[51]$ \\
\hline Maternal high school or greater degree & $193 / 311[62]$ & $263 / 402[65]$ \\
\hline Maternal asthma $(+)$ & $77 / 311[25]$ & $86 / 416[21]$ \\
\hline Prenatal ETS exposure $^{\mathrm{d}}(+)$ & $100 / 311[32]$ & $146 / 406[36]$ \\
\hline Postnatal ETS exposure $^{\mathrm{e}}(+)$ & $142 / 311[46]$ & $136 / 353$ [39] \\
\hline Seroatopy $^{\mathrm{f}}(+)$ & $89 / 311[29]$ & $46 / 186[25]$ \\
\hline Total $\operatorname{IgE}(\geq 50 \mathrm{IU} / \mathrm{mL})$ & $136 / 291[47]$ & $62 / 175[35]$ \\
\hline Current asthmag $^{\mathrm{g}}(+)$ & $61 / 311[20]$ & $33 / 182[18]$ \\
\hline Doctor diagnosed asthma $(+)$ & $86 / 311[28]$ & $50 / 182[28]$ \\
\hline Asthma medication use $(+)$ & $87 / 311[28]$ & $42 / 182[23]$ \\
\hline Recent wheeze $^{\mathrm{h}}(+)$ & $87 / 311[28]$ & $40 / 182[22]$ \\
\hline Body mass index $z$ score, median $[\mathrm{SD}]$ & $0.63[1.48]$ & $0.85[1.33]$ \\
\hline Underweight ( $<5$ th percentile) & $22 / 311[7]$ & $9 / 188[5]$ \\
\hline Normal weight ( 5 th to $<85$ th percentile) & $172 / 311[55]$ & $98 / 188[52]$ \\
\hline Overweight (85th to $<95$ th percentile) & $54 / 311[17]$ & $35 / 188[19]$ \\
\hline Obesity ( $\geq 95$ th percentile) & $63 / 311[20]$ & $46 / 188[25]$ \\
\hline Air monitor set up at age 5 years ${ }^{j}$ & $224 / 311[72]$ & $63 / 97[65]$ \\
\hline Prenatal pyrene median [range], ng/m³ & $2.53[0.77-17.2]$ & $2.68[0.28-97.0]$ \\
\hline
\end{tabular}

${ }^{a}$ None of the demographics differed significantly between the children included when compared to those excluded.

${ }^{\mathrm{b}}$ Includes participants who underwent residential monitoring and had questionnaire data collected at age 5 years.

c Due to missing data. $N=319$ CCCEH subjects did not undergo childhood air monitoring study, $n=35$ had no BMI data available, $n=10$ had no questionnaire data obtained, $n=28$ had invalid prenatal or age 5 PAH data, $n=4$ had missing covariates, $n=19$ had missing specific indoor IgE data and $n=2$ had invalid BC and PM 2.5 data.

d Prenatal ETS exposure was defined as the report of any smoker in the house during pregnancy.

e Postnatal ETS was defined as the report of any smoker in the house when the child was aged 6 months, 1, 2, 3 or 5 years.

${ }^{\mathrm{f}}$ At least one specific $\mathrm{IgE} \geq 0.35 \mathrm{IU} / \mathrm{mL}$ to any of five indoor allergens tested at age 5 .

${ }^{g}$ Current asthma defined as parental report of doctor diagnosis of asthma and taking asthma medication in the last 12 months at age 5 .

${ }^{\mathrm{h}}$ Recent wheeze defined as any report of wheeze in the past 12 months at age 5.

${ }^{\mathrm{i}}$ Body mass index (BMI) calculated by weight $(\mathrm{kg}) /$ height $(\mathrm{m})^{2}$, SD, standard deviation.

${ }^{\mathrm{j}}$ Whether residential monitoring conducted at age 5 vs. 6 years.

effect modification by obesity remained at both age 5 and 7 (e.g., RR [95\% CI] for 1-methylphenanthrene: 2.73 [1.09-6.79] in obese but not non-obese 5 years old with $P_{\text {interaction }}=0.004$ ). There were modest effect modifications of the association between asthma and 1-methylphenanthrnee, 3,6-dimethylphenanthrene, and pyrene by obesity after the exclusion of the upper or lower $5 \%$ of measures $\left(P_{\text {interaction }}<0.10\right)$. Lastly, after removing those who took inhaled corticosteroids $(n=19)$, the associations displayed in Figs. 2 and 3 remained, with a smaller RR (e-Fig. 1).

\section{Discussion}

We found that higher concentrations of age 5-6 methylphenanthrenes were associated with asthma among obese children, but not non-obese children, at ages 5 and 7 years. This is the first study to report that obesity modified the effect of age 5-6 years $\mathrm{PAH}$ concentrations on asthma in an urban cohort of young children.

The strengths of the study include its ability to control key risk factors for childhood asthma development such as seroatopy and prenatal PAH concentrations. The prenatal period is an important time window of exposure that may affect children's respiratory health and obesity in later life (Miller et al., 2004; Rundle et al., 2012). Another was the direct measurement of each child's exposure based on two-week integrated home indoor samples. Our previous studies showed that PAH levels, measured in the sequential two-week residential indoor samples over 6-8 weeks of sampling periods, are relatively stable and highly correleated with each other (Rundle et al., 2012), implying that a single measure of two-week averaged PAH levels may be considered an indicator of longer-term exposure at age 5-6 years. We also controlled for the association of other indoor air pollutants such as $\mathrm{PM}_{2.5}$ and $\mathrm{BC}$, previously associated with an increased risk of developing new wheeze among children aged 5-7 years in our cohort (Jung et al., 2012a).

We suspect substantial indoor emissions as the main sources of these semivolatile PAH (Jung et al., 2010a). These may include cigarette smoking, incense burning/candling, cooking and space heating (Gustafson et al., 2008; Jung et al., 2010a), and evaporation from petrochemicals used indoors such as hair spray, deodorant, hand lotion, shampoo, perfumes, vinyl siding, floor polish, shower curtains, insect repellent, and pesticides (Greeniacs, 2012). Certainly infiltration of outdoor-originated semivolatile $\mathrm{PAH}$ from petrogenic and pyrogenic emissions related to traffic and heating sources may contribute to the indoor measures as well (Jung et al., 2010a).

The primary finding suggests that obesity modifies the effects of age 5-6 years PAH exposure on asthma at age 5. We previously reported that age 5-6 years PAH concentrations, only when combined with high prenatal PAH measures, was associated with asthma among young children, suggesting that age 5-6 years PAH exposure alone is not a risk factor for adverse respiratory health. Additionally, a large body of epidemiologic data suggests that obesity is a risk factor for asthma (Castro-Rodriguez et al., 2001; Gilliland et al., 2003; Visness et al., 2010). Thus, these evidences lend support to the hypothesis that exposure to PAH and childhood obesity may have additive or synergistic effects on developing or worsening asthma. After controlling for prenatal PAH concentrations and other indoor air pollutants, we found that the risk of developing asthma increased with higher levels of methylphenanthrenes among obese, but not non-obese children, suggesting a synergistic effect of methylphenanthrenes and obesity. Significant interactions were replicated in an adjusted analysis controlling for maternal BMI, which previously was reported to 


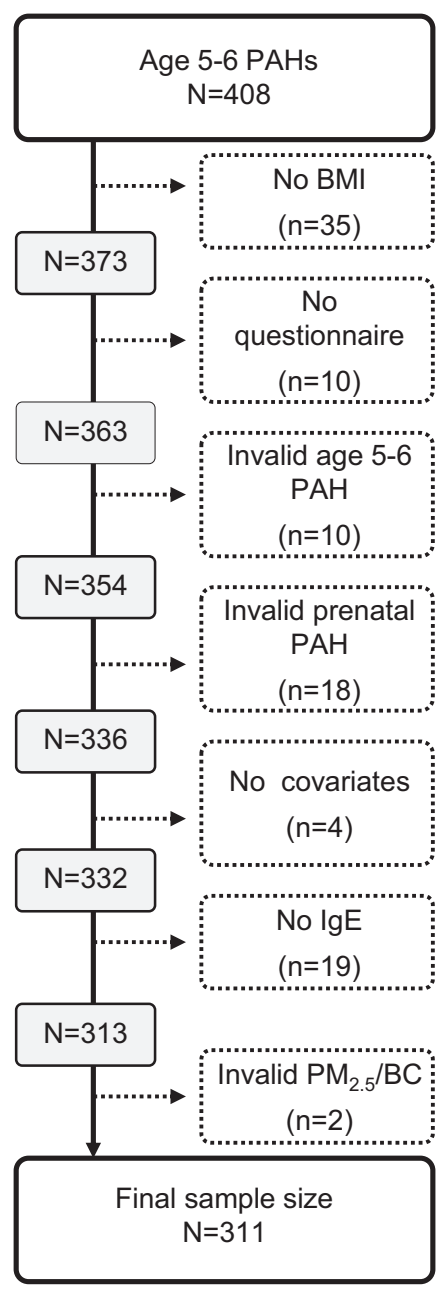

Fig. 1. Schematic demonstration of study data structure.

be a risk factor for childhood overweight or obesity (Gibson et al., 2007). Our finding was supported further by the RERI analyses showing a significant greater than additive interaction. We did not observe a main effect of obesity on asthma, possibly due to the fairly small sample size compared to other studies that have examined the association or differences in cohort characteristics (Castro-Rodriguez et al., 2001; Gilliland et al., 2003; Visness et al., 2010). A similar effect modification by obesity was found at a later age, suggesting that the effects of PAH-obesity on asthma may persist through age 7 . However, given the wide confidnece intervals seen at age 7 , these results should be interpreted with caution.

Additionally, the results from the tertile analysis of 9-methylphenanthrene and 3,6-dimethylphenanthrene conincided well with the main findings (e-Table 3 ), providing further confidence that the previously observed assocations were not driven by outliers. Nevertheless, the second tertile of other semivolatile $\mathrm{PAH}$ concentrations (e.g., phenanthrene, 1-methylphenenthrene, 2-methylphenanthrene), but not the third tertile, compared with the first tertile of concentration, was significantly associated with asthma in obese children, suggesting a non-linear dose-response relationship. We have no clear explanation for this but it could be partly due to the complexity of PAH mixtures relating to differences in volatility, bioaccumulation, toxicity and emission sources among individual components (National Park Service, 2013; Saha et al., 2009; Wolinska et al., 2011). Moreover, given the positive association between repeated pyrene exposure during prenatal and early childhood and asthma in our previous publication (Jung et al., 2012b), we reexamined for an interaction between repeated pyrene and obesity on asthma; no significant interaction was found in these analyses (data not shown). Our results also suggested a significant positive interaction between exposure to methylphenanthrenes and obesity on asthma that was more pronounced among nonatopic children (e-Table 4) or girls (e-Table 5). This observation is supported by other epidemiological findings showing air pollution (e.g. elemental carbon, particulate matter PAH)-associated asthma (Epstein et al., 2012; Jung et al., 2012b) and obesity-associated asthma (Gilliland et al., 2003; Visness et al., 2010) is more common in nonatopic individuals. Thus, we speculate that PAH-obesity related asthma may not necessarily be mediated by allergic inflammation but that other nonallergic pathways may play an important role. Also, our findings seem consistent with those from the longitudinal Tucson Children's Respiratory Study that indicated that the association between overweight/obesity and respiratory symptoms was largely restricted to school-age girls (CastroRodriguez et al., 2001).

Few cross-sectional studies have attempted to investigate effect modification of the association between air pollution and asthma by obesity. In one study of 904 elderly men, 48-hour higher levels of $\mathrm{O}_{3}$ were associated with a greater decline in $\mathrm{FEV}_{1}$ in the obese than in the non-obese (Alexeeff et al., 2007). A recent study with asthmatic individuals 65 years or older also reported that the mean daily concentration of elemental carbon (EC) was associated with poorer asthma control based on Asthma Control Questionnaire (ACQ) scores among obese, but not among non-obese asthmatics, suggesting that obesity may modify the effect of EC on asthma exacerbations (Epstein et al., 2012). Similarly, in a study with urban asthmatic children, overweight or obese children had increased odds of respiratory symptoms associated with higher levels of indoor $\mathrm{PM}_{2.5}$ and nitrogen dioxide $\left(\mathrm{NO}_{2}\right)$ ( Lu et al., 2013). Our study adds new evidence to support that obese young children are more prone to the effects of airborne PAH, methylphenanthrenes in particular, that are mostly generated from indoor sources on asthma at ages 5 and 7 years.

Despite these epidemiologic observations, the responsible biological mechanisms are not well-understood. A recent study conducted in mice has suggested that inhibition of the adipose derived hormone important in appetite and metabolism, leptin, increases parasympathetic signaling through the $\mathrm{M}_{3}$ muscarinic receptor in airway smooth muscle cells (Arteaga-Solis et al., 2013). This mechanism may thereby decrease bronchial diameter without affecting local inflammation in the bronchi, leading to bronchoconstriction and airway obstruction that could worsen in the setting of other asthma triggers like air pollution (Arteaga-Solis et al., 2013).

An alternative explanation may be that a sedentary lifestyle in obese children effectively indicates more time spent indoors where substantial semivolatile PAH sources are present, thereby increasing their exposure to asthma-promoting PAH. While this particular study was not designed to measure time indoors, other studies have shown that children at ages 5-7 years spend 65-68\% of their time indoors at home (Hubal et al., 2000), and obese children tend to be physically less active and possess a more sedentary lifestyle with excessive television watching or video game use (Tremblay and Willms, 2003). Also, in an animal study, Shore and colleagues reported that obese mice inhaled the greater dose of $\mathrm{O}_{3}$ to the lungs than normal-weight mice due to higher breathing frequency (Shore et al., 2003). Therefore, not only increased time spent indoors but also higher breathing frequency may lead obese children to be exposed to greater doses of asthmapromoting methylphenanthrenes, compared to non-obese children.

A significant association of $\mathrm{PAH}$ with asthma but not recent wheeze among obese children was observed. One interpretation is that asthmatic children using asthma medications (inhaled corticosteroids, beta 2 adrenergic agonists) would be less symptomatic following air pollution exposure and report less wheeze and 
Table 2

Associations between age 5-6 years PAH concentrations and asthma at age 5 years: effect modification by obesity.

\begin{tabular}{|c|c|c|c|c|}
\hline \multirow[b]{3}{*}{ Age 5-6 PAH $\left[\mathrm{IQR}_{\mathrm{ln}}\right]$} & \multirow{2}{*}{$\begin{array}{l}\text { Total sample main effect } \\
(n=311)\end{array}$} & \multicolumn{2}{|c|}{ Effect of age 5-6 PAH: stratified by obesity } & \multirow{2}{*}{$\frac{\text { Multiplicative interaction model }^{\mathrm{c}}}{(n=311)}$} \\
\hline & & Obese $(n=63)$ & Non-obese $(n=248)$ & \\
\hline & Adjusted $^{\mathrm{a}}$ RR [95\% CI] & Adjusted $^{\mathrm{a}}$ RR [95\% CI] & Adjusted $^{\mathrm{a}}$ RR [95\% CI] & $p$-Value for interaction \\
\hline Phe [0.77] & $0.89[0.67-1.17]$ & $0.83[0.48-1.44]$ & $0.80[0.58-1.11]$ & 0.073 \\
\hline Obesity & $1.18[0.68-2.04]$ & & & \\
\hline 1-Meph [0.76] & $1.09[0.80-1.48]$ & 2.62* $[1.17-5.88]$ & $0.92[0.65-1.31]$ & 0.012 \\
\hline Obesity & $1.20[0.70-2.06]$ & & & \\
\hline 2-Meph [0.73] & $0.98[0.74-1.30]$ & $0.93[0.46-1.87]$ & $0.92[0.68-1.23]$ & 0.285 \\
\hline Obesity & $1.18[0.69-2.03]$ & & & \\
\hline 3-Meph [0.74] & $0.99[0.76-1.30]$ & $1.27[0.67-2.42]$ & $0.92[0.68-1.23]$ & 0.090 \\
\hline Obesity & $1.18[0.69-2.04]$ & & & \\
\hline 9-Meph [0.73] & $1.02[0.77-1.35]$ & 2.17* $[1.06-4.45]$ & $0.91[0.69-1.20]$ & 0.028 \\
\hline Obesity & $1.19[0.69-2.05]$ & & & \\
\hline 1,7-DMeph [0.71] & $0.94[0.73-1.22]$ & $1.05[0.69-1.61]$ & $0.92[0.65-1.28]$ & 0.573 \\
\hline Obesity & $1.15[0.66-2.03]$ & & & \\
\hline 3,6-DMeph [0.71] & $1.02[0.73-1.41]$ & $\mathbf{2 . 6 9} *[1.21-5.94]$ & 0.88 [0.60-1.29] & 0.077 \\
\hline Obesity & $1.19[0.69-2.05]$ & & & \\
\hline Pyrene [0.56] & $0.88[0.67-1.16]$ & $0.93[0.44-1.95]$ & 0.81 [0.59-1.12] & 0.025 \\
\hline Obesity & 1.19 [0.69-2.06] & & & \\
\hline$\sum_{8} \mathrm{PAH}_{\text {semivolatile }}[0.74]$ & $0.91[0.69-1.20]$ & 0.87 [0.50-1.53] & $0.82[0.60-1.12]$ & 0.069 \\
\hline Obesity & 1.18 [0.68-2.03] & & & \\
\hline$\sum_{8} \mathrm{PAH}_{\text {nonvolatile }}[1.02]^{\mathrm{b}}$ & $0.81[0.54-1.23]$ & 0.84 [0.33-2.09] & $0.74[0.46-1.18]$ & 0.089 \\
\hline Obesity & $1.10[0.62-1.94]$ & & & \\
\hline
\end{tabular}

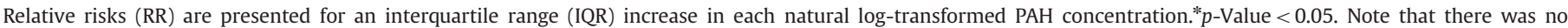
observed association between PAHs and recent wheeze in stratified models by presence/absence of obesity.

${ }^{a}$ Model adjusted for ethnicity, sex, maternal education, maternal asthma, prenatal ETS, postnatal ETS, cold/flu season, residential monitoring conducted at age 5 vs.

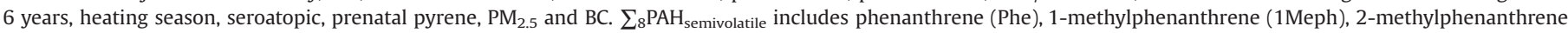

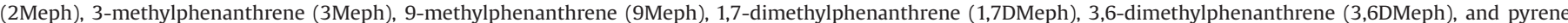

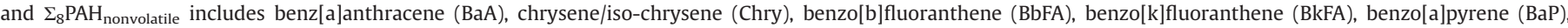
indeno[1,2,3-c,d]pyrene (IP), dibenz[a,h]anthracene (DahA), and benzo[g,h,i]perylene (BghiP).

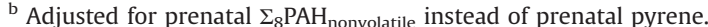

${ }^{c}$ A multiplicative interaction term was included in adjusted model.

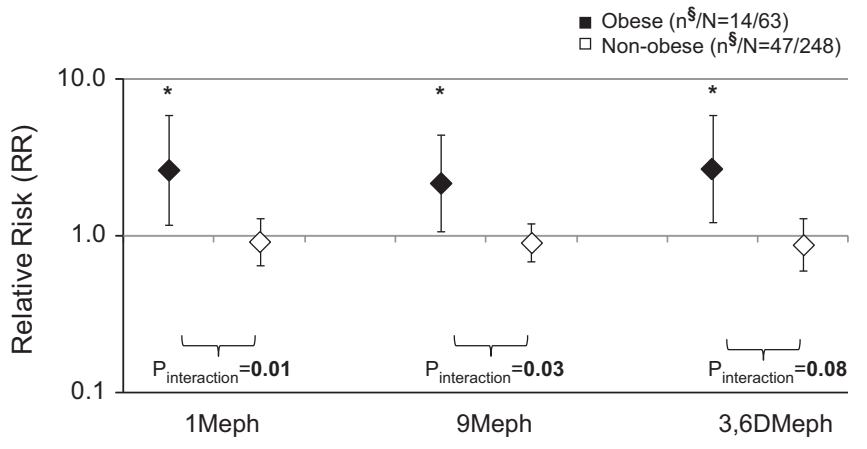

Fig. 2. Effect modification of obesity on the association between childhood PAH measures and current asthma at age 5 years. $n^{\S}$ : Cases of current asthma and $N$ : total sample size; relative risk estimates of asthma for an interquartile increase in $\log$ 1-methylphenanthrene (1Meph), 3-methylphenanthrene (3Meph), and 3,6dimethylphenanthrene (3,6DMeph) concentrations among obese $(n=63)$ and nonobese $(n=248)$ children, adjusting for ethnicity, sex, maternal education, maternal asthma, prenatal ETS, childhood ETS, cold/flu season, residential monitoring conducted at age 5 vs. 6 years, heating season, seroatopic, prenatal pyrene, $\mathrm{PM}_{2.5}$ and BC; $P_{\text {interaction: }} p$-values for multiplicative interaction; ${ }^{*} p<0.05$.

potentially bias the results. Sensitivity analyses for children without ICS revealed that a positive interaction remained although the associations between exposure to methylphenanthrenes and asthma were somewhat weakened (changes in RR ranged between $17 \%$ and 24\%) among obese children, compared to those reported in main analysis.

We acknowledge several limitations. First, the sample size is fairly small, especially for stratified analyses by obesity status. Second, many analyses were conducted on children with asthma diagnosed through age 5 , as opposed to incident asthma at age 5 .

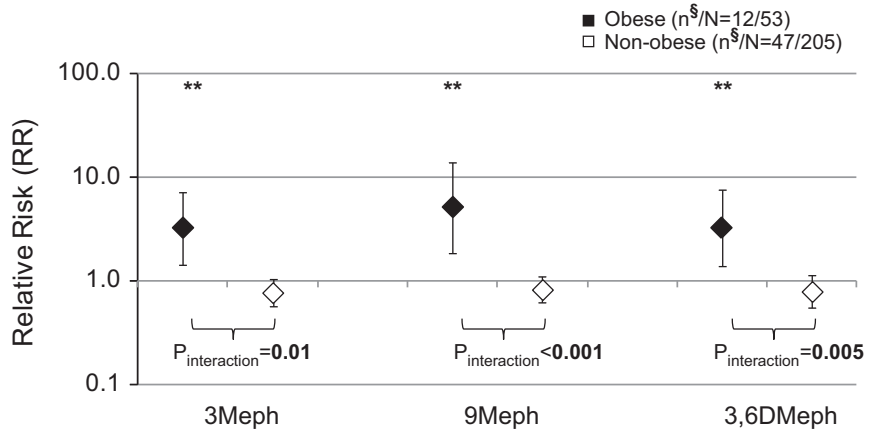

Fig. 3. Effect modification of obesity on the association between childhood $P A H$ exposure and current asthma at age 7 years. $n^{\S}$ : Cases of current asthma and $N$ : total sample size; relative risk estimates of asthma for an interquartile increase in log 3-methylphenanthrene (3Meph), 9-methylphenanthrene (9Meph), and 3,6dimethylphenanthrene (3,6DMeph) concentrations among obese $(n=53)$ and nonobese $(n=205)$ children, adjusting for ethnicity, sex, maternal education, maternal asthma, prenatal ETS, childhood ETS, cold/flu season, residential monitoring conducted at age 5 vs. 6 years, heating season, seroatopic, prenatal pyrene, $\mathrm{PM}_{2.5}$ and BC; $P_{\text {interaction }} p$-values for multiplicative interaction; $* * p<0.01$.

To account for the temporality between the exposure and the outcome, we performed sensitivity analyses that excluded children with a preexisting doctor diagnosis of asthma before age 5 . A significant interaction (PAH by obesity) was detected for most of the semivolatile PAH (e-Table 6), although the statistical power of the stratified analyses was limited. Also, only $72 \%$ of residential monitorings were conducted at age 5 years when asthma outcomes were measured. However, we controlled for age at time of indoor monitoring in the model to address this latter limitation. While the results were quite consistent when obesity was replaced 
Table 3

$P$ value for positive ${ }^{a}$ interactions between age 5-6 years PAH concentrations and body mass index (BMI) $)^{\mathrm{b}} z$ score on asthma at ages 5 and 7 years.

\begin{tabular}{llc}
\hline & \multicolumn{2}{l}{$p$-Value for interaction ${ }^{\mathrm{c}}(\mathrm{PAH} \times \mathrm{BMI} z$ score $)$ on asthma } \\
\cline { 2 - 3 } 5 Year $(n=311)$ & 7 Year $(n=258)$ \\
\hline Phe & $\mathbf{0 . 0 1 8}$ & $\mathbf{0 . 0 0 8}$ \\
1-Meph & $\mathbf{0 . 0 2 4}$ & $\mathbf{0 . 0 2 5}$ \\
2-Meph & $\mathbf{0 . 0 3 2}$ & $\mathbf{0 . 0 1 3}$ \\
3-Meph & $\mathbf{0 . 0 2 5}$ & $\mathbf{0 . 0 0 3}$ \\
9-Meph & $\mathbf{0 . 0 0 7}$ & $\mathbf{0 . 0 0 1}$ \\
1,7-DMeph & 0.159 & 0.254 \\
3,6-DMeph & $\mathbf{0 . 0 1 7}$ & $\mathbf{0 . 0 1 6}$ \\
Pyrene & 0.220 & 0.146 \\
$\sum_{8}$ PAH $_{\text {semivolatile }}$ & $\mathbf{0 . 0 1 2}$ & $\mathbf{0 . 0 0 5}$ \\
$\sum_{8}$ PAH $_{\text {nonvolatile }}$ & 0.649 & 0.703
\end{tabular}

a Positive interaction defined as RR for multiplicative interaction greater than 1

${ }^{\mathrm{b}}$ Weight $(\mathrm{kg}) /$ height $(\mathrm{m})^{2}$.

${ }^{c}$ Multiplicative interaction model adjusted for ethnicity, sex, maternal education, maternal asthma, prenatal ETS, postnatal ETS, cold/flu season, residential monitoring conducted at age 5 vs. 6 years, heating season, seroatopic, prenatal

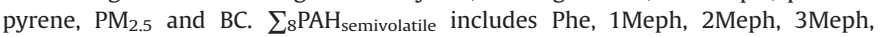

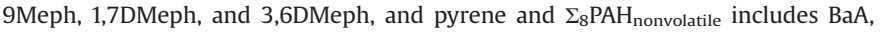
Chry, BbFA, BkFA, BaP, IP, DahA, and BghiP.

${ }^{\mathrm{d}}$ Adjusted for prenatal $\Sigma_{8} \mathrm{PAH}_{\text {nonvolatile }}$ instead of prenatal pyrene.

with continuous BMI $z$ score, an interaction effect was attenuated after the exclusion of the upper or lower $5 \%$ of PAH measures. Lastly, collection of physical time-activity information during sampling periods would have helped assess whether sedentary lifestyle or increased time spent indoors contributed significantly.

\section{Conclusion}

This study offers the first evidence that obesity may modify the effect of age 5-6 years PAH exposure, and the semivolatile methylphenanthrenes in particular, on asthma in young children at ages 5 and 7 years. These results suggest that obesity may enhance the vulnerability to effects of age 5-6 years PAH exposure on airway outcomes, particularly for nonatopic children and for girls. Specific interventions aimed at reducing PAH exposure and preventing excessive weight gain or increasing vigorous physical activity for young children living in the inner city should be evaluated as valuable tools in preventing adverse respiratory outcomes during childhood.

\section{Acknowledgments}

This work was supported by NIH (R01ES013163, P50ES015905, P01ES09600, R01ES08977, P30ES09089), EPA (R827027, RD832141, RD834509), the Educational Foundation of America, the John \& Wendy Neu Family Foundation, the New York Community Trust, and the Trustees of the Blanchette Hooker Rockefeller Fund.

\section{Appendix. Supplementary information}

Supplementary data associated with this article can be found in the online version at http://dx.doi.org/10.1016/j.envres.2013.12.002.

\section{References}

Akinbami, L.J., et al., 2009. Status of childhood asthma in the United States, 19802007. Pediatrics 123, S131-S145.
Alexeeff, S.E., et al., 2007. Ozone exposure and lung function. Chest 132, 1890-1897. Arteaga-Solis, E., et al., 2013. Inhibition of leptin regulation parasympathetic signaling as a cause of extreme body weight associated asthma. Cell Metab. $17,35-48$.

Bonner, S., et al., 2006. Validating an asthma case detection instrument in a Head Start sample. J. Sch. Health 76, 471-478.

CDC, 2011. Asthma prevalence, disease characteristics, and self-management education - United States, 2001-2009. Centers for disease control and prevention. Morb. Mortal. Wkly. Rep. (MMWR) 60 (17), 547-552.

Castro-Rodriguez, J.A., et al., 2001. Increased incidence of asthmalike symptoms in girls who become overweight or obese during the school years. Am. J. Respir. Crit. Care Med. 163, 1344-1349.

Centers for Disease Control and Prevention, 2004. A SAS Program for the CDC Growth Charts. Centers for Disease Control and Prevention, Atlanta, GA.

Donohue, K.M., et al., 2008. Anti-cockroach and anti-mouse IgE are associated with early wheeze and atopy in an inner-city birth cohort. J. Allergy Clin. Immunol. $122,914$.

Epstein, T.G., et al., 2012. Poor asthma control and exposure to traffic pollutants and obesity in older adults. Ann. Allergy, Asthma Immunol. 108, 423-428.

Gibson, L.Y., et al., 2007. The role of family and maternal factors in childhood obesity. Med. J. Aust. 186, 591.

Gilliland, F.D., et al., 2003. Obesity and the risk of newly diagnosed asthma in school-age children. Am. J. Epidemiol. 158, 406-415.

Greeniacs, 2012. Petroleum Product. Available from: 〈http://www.greeniacs.com/ GreeniacsArticles/Energy/Petroleum-Products.html $\rangle$ (accessed 22.10.12).

Gustafson, P., et al., 2008. Indoor levels of polycyclic aromatic hydrocarbons in homes with or without wood burning for heating. Environ. Sci. Technol. 42, 5074-5080.

Hubal, E.A.C., et al., 2000. Children's exposure assessment: a review of factors influencing children's exposure, and the data available to characterize and assess that exposure. Environ. Health Perspect. 108, 475.

Jenkins, M.A., et al., 1996. Validation of questionnaire and bronchial hyperresponsiveness against respiratory physician assessment in the diagnosis of asthma. Int. J. Epidemiol. 25, 609.

Johnston, R.A., et al., 2008. Diet-induced obesity causes innate airway hyperresponsiveness to methacholine and enhances ozone-induced pulmonary inflammation. J. Appl. Physiol. 104, 1727-1735.

Jung, K.H., et al., 2010a. Effects of heating season on residential indoor and outdoor polycyclic aromatic hydrocarbons, black carbon, and particulate matter in an urban birth cohort. Atmos. Environ. 44, 4545-4552.

Jung, K.H., et al., 2010b. Assessment of benzo (a) pyrene-equivalent carcinogenicity and mutagenicity of residential indoor versus outdoor polycyclic aromatic hydrocarbons exposing young children in New York City. Int. J. Environ. Res. Public Health 7, 1889-1900.

Jung, K.H., et al., 2012a. Childhood exposure to fine particulate matter and black carbon and the development of new wheeze between ages 5 and 7 in an urban prospective cohort. Environ. Int. 45, 44-50.

Jung, K.H., et al., 2012b. Repeated exposure to polycyclic aromatic hydrocarbons and asthma: effect of seroatopy. Ann. Allergy, Asthma Immunol. 109, 249-254.

Lu, F.L., et al., 2006. Increased pulmonary responses to acute ozone exposure in obese db/db mice. Am. J. Physiol. - Lung Cell. Mol. Physiol. 290, L856-L865.

Lu, K.D., et al., 2013. Being overweight increases susceptibility to indoor pollutants among urban children with asthma. J. Allergy Clin. Immunol. 131, 1017-1023.

Miller, R.L., et al., 2004. Polycyclic aromatic hydrocarbons, environmental tobacco smoke, and respiratory symptoms in an inner-city birth cohort. Chest 126, 1071-1078.

National Park Service, 2013. Environmental Contaminants Encyclopedia Entry for 1-methylphenanthrene. Available from: 〈http://www.nature.nps.gov/hazards safety/toxic/phen1met.pdf (accessed 05.08.13).

Nicholas, S.W., et al., 2005. Addressing the childhood asthma crisis in Harlem: the Harlem children's zone asthma initiative. Am. J. Public Health 95, 245.

Rundle, A., et al., 2012. Association of childhood obesity with maternal exposure to ambient air polycyclic aromatic hydrocarbons during pregnancy. Am. J. Epidemiol. 175, 1163-1172.

Saha, M., et al., 2009. Sources of sedimentary PAHs in tropical Asian waters: differentiation between pyrogenic and petrogenic sources by alkyl homolog abundance. Mar. Pollut. Bull. 58, 189-200.

Shore, S.A., et al., 2003. Responses to ozone are increased in obese mice. J. Appl. Physiol. 95, 938-945.

Thorpe, L., et al., 2004. Childhood obesity in New York City elementary school students. Am. J. Public Health 94, 1496-1500.

Tremblay, M., Willms, J., 2003. Is the Canadian childhood obesity epidemic related to physical inactivity? Int. J. Obes. 27, 1100-1105.

Visness, C.M., et al., 2010. Association of childhood obesity with atopic and nonatopic asthma: results from the National Health and Nutrition Examination Survey 1999-2006. J. Asthma 47, 822-829.

Wolinska, L., et al., 2011. Preliminary study on adverse effects of phenanthrene and its methyl and phenyl derivatives in larval zebrafish, Danio rerio. Environ. Biotechnol. 7, 21-33.

Zou, G., 2004. A modified poisson regression approach to prospective studies with binary data. Am. J. Epidemiol. 159, 702-706. 